\title{
Decoherence and quantum quench: their relationship with excited state quantum phase transitions
}

\author{
J. E. García-Ramos*, J. M. Arias ${ }^{\dagger}$, P. Cejnar**, J. Dukelsky, P. \\ Pérez-Fernández ${ }^{\S}$ and A. Relaño ${ }^{\mathbb{I}}$ \\ *Departamento de Física Aplicada, Universidad de Huelva, 21071 Huelva, Spain \\ ${ }^{\dagger}$ Departamento de Física Atómica, Molecular y Nuclear, Facultad de Física, Universidad de \\ Sevilla, Apartado 1065, 41080 Sevilla, Spain \\ ** Institute of Particle and Nuclear Physics, Faculty of Mathematics and Physics, Charles \\ University, V Holešovičkách 2, Prague, 18000, Czech Republic \\ ${ }^{*}$ Instituto de Estructura de la Materia, CSIC, Serrano 123, E-28006 Madrid, Spain \\ ${ }^{\S}$ Depart. de Física Aplicada III, Escuela de Ingenieros, Univ. de Sevilla, 41092 Sevilla, Spain \\ "Depart. de Física Aplicada I and GISC, Univ. Complutense de Madrid, 28040 Madrid, Spain
}

\begin{abstract}
We study the similarities and differences between the phenomena of Quantum Decoherence and Quantum Quench in presence of an Excited State Quantum Phase Transition (ESQPT). We analyze, on one hand, the decoherence induced on a single qubit by the interaction with a two-level boson system with critical internal dynamics and, on the other, we treat the quantum relaxation process that follows an abrupt quench in the control parameter of the system Hamiltonian. We explore how the Quantum Decoherence and the quantum relaxation process are affected by the presence of an ESQPT. We conclude that the dynamics of the qubit or the quantum relaxation process change dramatically when the system passes through a continuous ESQPT.
\end{abstract}

Keywords: Quantum decoherence, quantum quench, excited state quantum phase transition PACS: 03.65.Yz, 05.70.Fh, 64.70.Tg

\section{INTRODUCTION}

Quantum Decoherence (QD) and Quantum Quench (QQ) are two, apparently, disconnected phenomena; one related with the lost of quantum information and the other involved with non equilibrium phenomena in interacting quantum systems. A Quantum Phase Transition (QPT), or its version for excited states, is an abrupt change in the structure of the ground state of the system under a small variation of the control parameter of the Hamiltonian. This contribution is devoted to the study of the relationship between this three quantum phenomena.

Real quantum systems always interact with the environment. This interaction leads to QD, the process by which quantum information is degraded and purely quantum properties of a system are lost [1]. From a fundamental point of view, QD provides a theoretical basis for the quantum-classical transition [1], emerging as a possible explanation of the quantum origin of the classical world. From a practical point of view, it is a major obstacle for building a quantum computer [2] since it can produce the loss of the quantum character of the computer. Therefore, a complete characterization of the QD process and its relation with the physical properties of the system and the environment is needed for 
both fundamental and practical purposes.

A QQ represents an abrupt, diabatic change $\lambda_{1} \rightarrow \lambda_{2}$ of the control parameter followed by a system-specific quantum relaxation process. Pioneering theoretical works in this field appeared already in the late 1960s [3], but a really rapid growth of interest was triggered by experimental studies at the beginning of this millennium [4].

A QPT is a sudden change of the ground-state structure at a certain critical value of the control parameter $\lambda$. It can be observed as a nonanalytic evolution of the system's energy and wave function induced by an adiabatic variation of the control parameter $\lambda$ across the quantum critical point $\lambda_{c 0}$ at zero temperature. First discussed in the $1970 \mathrm{~s}$ [5], the QPT phenomena become very important in the context of solid state physics [6] as well as in nuclear and many-body physics—see, e.g., recent reviews [7, 8]. An Excited State Quantum Phase Transition (ESQPT) [9] is analogous to a standard QPT, but taking place in some excited state of the system, which defines the critical energy $E_{c}$ at which the transition takes place. We can distinguish between different kinds of ESQPT, either first order or continuous [10]. In this contribution we will mainly concentrate in the latter case, which usually entails a singularity in the density of states (for an illustration of continous ESQPT see Fig. 1). We will see along this contribution how QD and QQ are enhanced due to the existence of an ESQPT.

\section{CONNECTION BETWEEN DECOHERENCE AND ESQPT}

The connection between decoherence and environmental QPTs has been recently investigated in [11]. In this contribution and in references [12,13] we analyze the relationship between decoherence and an environmental ESQPT.

Here, we consider an environment having both QPTs and ESQPTs coupled to a single qubit. The Hamiltonian of the environment, defined as a function of a control parameter $\alpha$, presents a QPT at a critical value $\alpha_{c}$. We define a coupling between the central qubit and the environment that entails an effective change in the control parameter, $\alpha \rightarrow \alpha^{\prime}$, making the environment to cross the critical point if $\alpha^{\prime}>\alpha_{c}$. Moreover, the coupling also implies an energy transfer to the environment $E \rightarrow E^{\prime}$, and therefore it can also make the environment to reach the critical energy $E_{c}$ of an ESQPT.

Following [11] we will consider our system composed by a spin $1 / 2$ particle coupled to a bosonic environment by the Hamiltonian $H_{S E}$ :

$$
H_{S E}=I_{S} \otimes H_{E}+|0\rangle\left\langle 0\left|\otimes H_{\lambda_{0}}+\right| 1\right\rangle\langle 1| \otimes H_{\lambda_{1}},
$$

where $|0\rangle$ and $|1\rangle$ are the two components of the spin $1 / 2$ system, and $\lambda_{0}, \lambda_{1}$ the coupling of each component with the environment. The three terms $H_{E}, H_{\lambda_{0}}$ and $H_{\lambda_{1}}$ act on the Hilbert space of the environment.

With this kind of coupling, the environment evolves with an effective Hamiltonian depending on the state of the central spin $H_{j}=H_{E}+H_{\lambda_{j}}, j=0,1$. If the environment is initially in its ground state $\left|g_{0}\right\rangle$, the decoherence factor is determined, up to an irrelevant phase factor, by $H_{1}$, and its absolute value is equal to

$$
|r(t)|=\left|\left\langle g_{0}\left|e^{-i H_{1} t}\right| g_{0}\right\rangle\right| .
$$




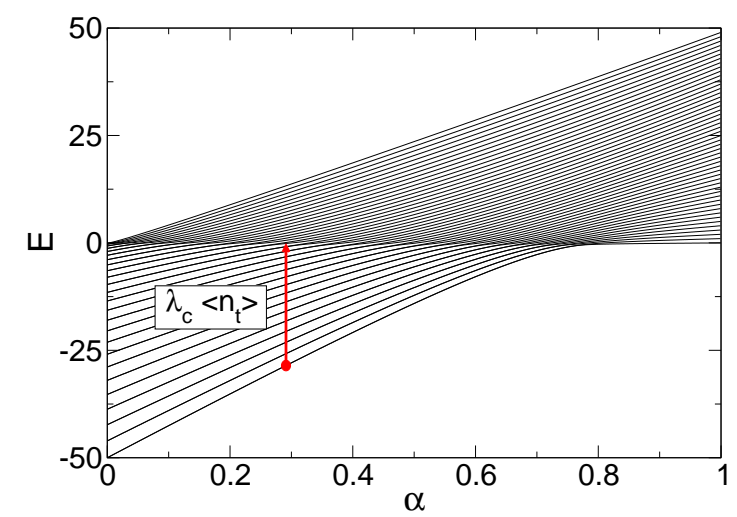

FIGURE 1. Energy levels for the environment Hamiltonian (3) with $L=0$ and $N=50$. The arrow shows the jump that produces in the environment the coupling with the central qubit.

A value of $|r(t)|$ equal to zero implies that the qubit is no longer in a superposition of states $|0\rangle+|1\rangle$.

To be specific, let us consider a two level boson Hamiltonian, constructed out of scalar bosons, $s$, in the lowest level and bosons carrying an arbitrary angular momentum $L$ in the upper level.

$$
H_{E}=\alpha n_{L}-\frac{1-\alpha}{N} Q^{\chi} \cdot Q^{\chi}, \text { with } Q_{\mu}^{\chi}=s^{\dagger} L+L^{\dagger} s+\chi\left[L^{\dagger} \times \tilde{L}\right]_{\mu}^{(L)},
$$

where $n_{L}$ is the number of $L$ bosons, $N$ the total number of bosons and $\cdot$ stands for the scalar product.

This Hamiltonian has a second order QPT at $\alpha_{c}=4 / 5$ for $\chi=0$ [14], while experiences a first order phase transition for $\chi \neq 0$. We will focus in the case of $\chi=0$. Using the coherent state formalism it can be shown that for $\alpha>4 / 5$ the environment is a condensate of $s$ bosons corresponding to a symmetric phase. For $\alpha<4 / 5$ the environment condensate mixes $s$ and $L$ bosons forming a non-symmetric phase.

Choosing $\lambda_{0}=0$ and $\lambda_{1}=\lambda$, the coupling Hamiltonian reduces to a very simple form $H_{\text {Coup }}=\lambda n_{L}$, which results into the effective Hamiltonians for each component of the systems, $H_{0}=H_{E}$ and $H_{1}=H_{E}(\alpha \rightarrow \alpha+\lambda)$. Therefore, the system-environment coupling parameter $\lambda$ modifies the environment Hamiltonian. It is straightforward to show that $H_{1}$ goes through a second order QPT at $\lambda_{*}=4-5 \alpha$, for $\alpha<4 / 5$, using [14]. Furthermore, a semiclassical calculation [9] shows that $H_{E}$ also passes through an ESQPT at $E_{c}=0$, if $\lambda<\lambda_{*}$. This phenomenon is illustrated in Fig. 1.

We start the evolution with the ground state of the environment $\left|g_{0}\right\rangle$. At $t=0$ we switch on the interaction between the system and the environment, and let the system evolve under the complete Hamiltonian. By instantaneously switching on this interaction, the energy of the environment increases, and its state gets fragmented into a region with average energy equal to $E=\left\langle g_{0}\left|H_{1}(\alpha)\right| g_{0}\right\rangle$. Therefore, if $\left\langle g_{0}\left|H_{1}(\alpha)\right| g_{0}\right\rangle=0$, the coupling with the central qubit induces the environment to jump into a region around the critical energy $E_{c}$. This is illustrated in Fig. 1. Starting from a state in the non-symmetric phase with $\alpha<\alpha_{c}$, the coupling with the qubit, $H_{\lambda_{1}}=\lambda n_{L}$ increases the energy of the environment up to the critical point $E_{c}$. Resorting to the coherent state approach, we can 


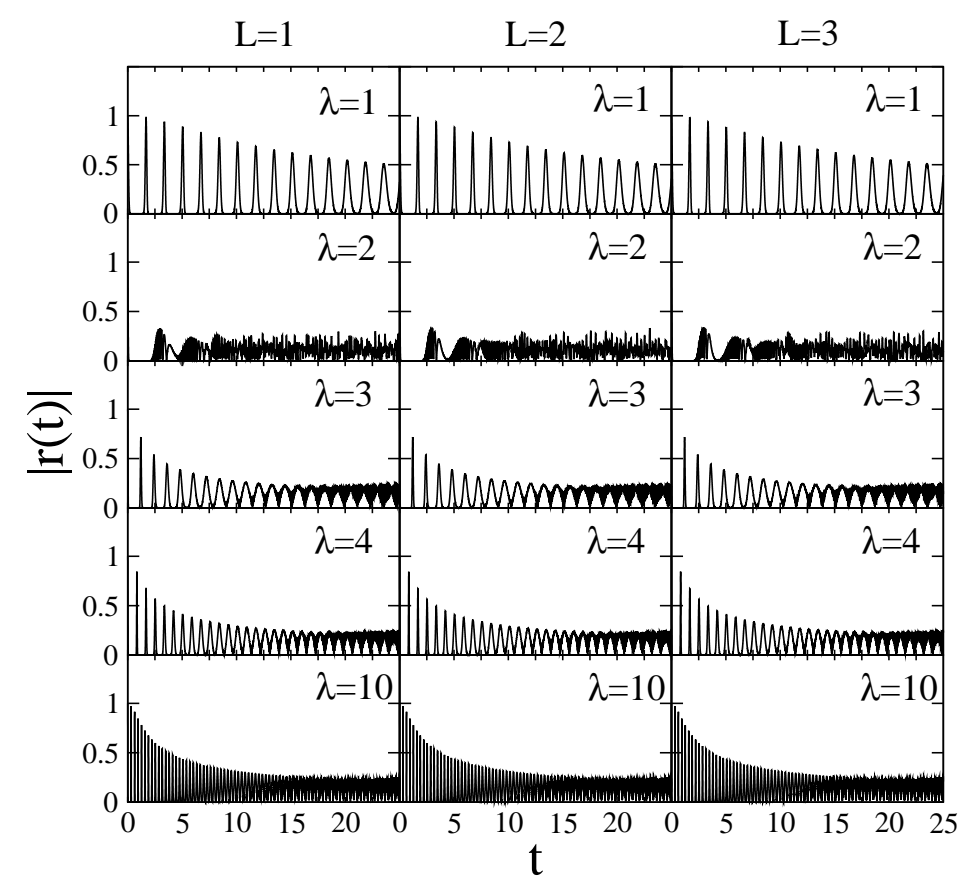

FIGURE 2. $|r(t)|$ for $\alpha=0$ and $\chi=0$, five different values of $\lambda$ and $L=1,2,3$. In all cases $N=1000$. The coupling constant $\lambda=2$ corresponds to the critical value (see eq. (4)).

obtain a critical value of the coupling strength

$$
\lambda_{c}(\alpha)=\frac{1}{2}(4-5 \alpha), \quad \alpha<\frac{4}{5}
$$

In Fig. 2 we show the modulus of the decoherence factor $|r(t)|$ for $\alpha=0$ and $\chi=0$ and several values of $\lambda$ and $L$, corresponding to the vibron $(L=1)$, the IBM $(L=2)$ and the octupole model $(L=3)$. First, we note that the presented behavior is independent on $L$. In four of the five cases of $\lambda$ we can see a similar pattern, fast oscillations plus a smooth decaying envelope. The most striking feature of Fig. 2 is the panel corresponding to $\lambda=2$, for which $|r(t)|$ quickly decays to zero and then randomly oscillates around a small value. We note that this particular case constitutes a singular point for both the shape of the envelope of $|r(t)|$ and the period of its oscillating part. Making use of Eq. (4) for $\alpha=0$ we obtain precisely $\lambda_{c}=2$, the value at which the coherence of the system is completely lost. Therefore, the existence of an ESQPT in the environment has a strong influence on the decoherence that it induces in the central system. We can summarize this result with the following conjecture:

If the system-environment coupling drives the environment to the critical energy $E_{c}$ of a continuous ESQPT, the decoherence induced in the coupled qubit is maximal.

This conjecture has been checked for different values of $\alpha<\alpha_{c}=4 / 5$ obtaining in all the cases that the rapid decay to zero of $|r(t)|$ always happens for $\lambda \approx \lambda_{c}$ (see Fig. 3 of reference [12]). It has been also checked how this magnitude behaves in the thermodynamical limit. The results displayed in Fig. 4 of reference [12] confirm that the presence of an ESQPT in the environment spectrum is clearly signaled by the qubit 
decoherence factor. Moreover, it can be defined an order parameter for the ESQPT related to $|r(t)|$.

To summarize, our main finding is that the decoherence is maximal when the systemenvironment coupling introduces in the environment the energy required to undergo a continuous ESQPT and that this results are independent on the value of $L$. Note that the conclusions obtained here are only valid in the case of continuous ESQPT. In the case of first order ESQPT, the decoherence is no longer affected by the presence of an ESQPT in the environment (see [13] for details).

\section{CONNECTION BETWEEN QUANTUM QUENCH AND ESQPT}

To study the QQ (see [15] for more details) we consider a simple composite system consisting of two interacting subsystems: i) a single bosonic mode written in terms of the creation and annihilation operators $b^{\dagger}$ and $b$ and therefore described by a HeisenbergWeyl algebra HW(1), and ii) a susbsystem described with a SU(1,1) algebra, written in terms of the operators $K_{ \pm}=K_{x} \pm i K_{y}$ and $K_{0}=K_{z}$, which verify

$$
\left[K_{0}, K_{ \pm}\right]= \pm K_{ \pm},\left[K_{+}, K_{-}\right]=-2 K_{0}
$$

The whole system may serve to describe the coexistence of two-atom molecules with dissociated atoms. Other similar models are the Jaynes-Cummings [16] and the Dicke [17] models which are based on a $\mathrm{HW}(1) \otimes \mathrm{SU}(2)$ algebra.

The total Hamiltonian to be considered reads as

$$
H=\omega_{0} K_{0}+\omega b^{\dagger} b+\frac{\lambda}{\sqrt{M}}\left[b K_{+}+b^{\dagger} K_{-}\right]
$$

where $\lambda / \sqrt{M} \geq 0$ is a scaled coupling parameter and $\omega, \omega_{0}$ stand for single-particle energies (we set $\hbar=1$ ).

Note that the $K$ operators can be written in terms of creation and annihilation operators

$$
K_{+}=\frac{1}{2}\left(a^{\dagger}\right)^{2}, K_{-}=\frac{1}{2} a^{2}, K_{0}=\frac{1}{2}\left(a^{\dagger} a+\frac{1}{2}\right) .
$$

Therefore, the $b$ bosons can be understood as representing two-atom molecules, while the $a$ bosons as representing single atoms.

$M$ is a conserved quantity which is connected with the number of two-atom molecules $\left(N_{b}\right)$ and the number of single atoms $\left(N_{a}\right)$. It reads as $M=2 N_{b}+N_{a}$, for even values of $N_{a}$ and as $M=2 N_{b}+N_{a}-1$ for odd values of $N_{a}$. Assuming $\omega_{0}>\omega$, the case $\lambda=0$ corresponds to a ground state which is a condensate of $b$ bosons: $\left|N_{b}=M / 2\right\rangle \otimes \mid N a=0$ or 1$\rangle$. However, for sufficiently large values of the coupling parameter $\lambda$, the interaction between the molecules and atomic pairs supports a more balanced distribution of the expectation values $\left\langle N_{a}\right\rangle$ and $\left\langle 2 N_{b}\right\rangle$.

A typical spectrum of the SU(1,1) is depicted in Fig. 3, where the evolution of quantum spectra is plotted as a function of the interaction parameter $\lambda$, showing clear indications of the ground-state QPT and its extension into the ESQPT on the right-hand 


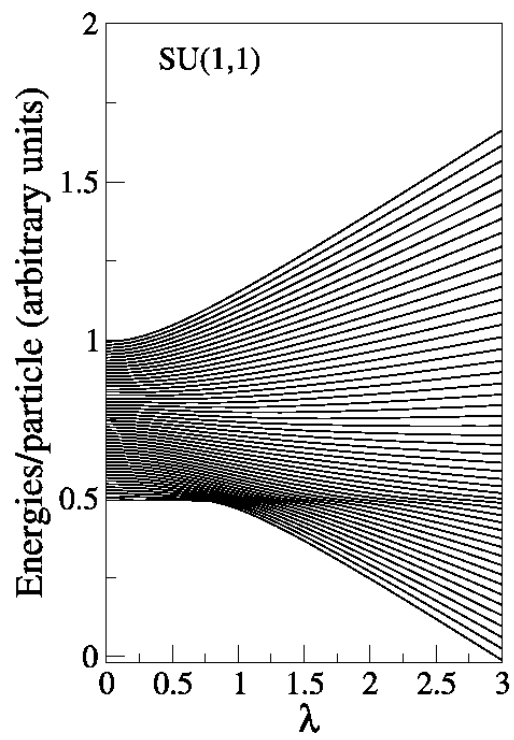

FIGURE 3. Level dynamics for the $\mathrm{SU}(1,1)$ model with $M=2000$ and $\Delta \omega=\omega_{0}-\omega=1$. The scaled energies were obtained by an exact diagonalization. The ESQPT above the ground state critical point $\lambda=0.707$ is apparent in the bunching of levels around critical energies $\mathscr{E}_{c}^{(1)}=0.5$.

side of the critical point. The calculation was done in a finite-size case, but it shows well pronounced precursors of the phase transitional behavior.

Suppose a system described trough a $S U(1,1)$ Hamiltonian that is initially prepared in the ground state $\left|\psi_{g s}\left(\lambda_{0}\right)\right\rangle \equiv\left|\psi_{0}\right\rangle$ of $\mathscr{H}\left(\lambda_{0}\right) \equiv \mathscr{H}_{0}$ with energy per particle $E_{0}\left(\lambda_{0}\right) / \aleph \equiv$ $\mathscr{E}_{0}$. At time $t=0$, the value of the control parameter is abruptly changed from $\lambda_{0}$ to $\lambda_{1}=$ $\lambda_{0}+\Delta$. The state $\left|\psi_{0}\right\rangle$ is no more an eigenstate of the new Hamiltonian $\mathscr{H}\left(\lambda_{1}\right) \equiv \mathscr{H}_{1}$ and starts evolving. The evolution in time $t>0$ can be monitored by a survival probability $p_{0}(t)=\left|a_{0}(t)\right|^{2}$, where

$$
a_{0}(t)=\left\langle\psi_{0}\left|e^{-i \mathscr{H}_{1} t}\right| \psi_{0}\right\rangle=\sum_{i}|\underbrace{\left\langle\mathscr{E}_{1 i} \mid \psi_{0}\right\rangle}_{c_{i}}|^{2} e^{-i \mathscr{E}_{1 i} t}=\sum_{i}\left|c_{i}\right|^{2} e^{-i \mathscr{E}_{1 i} t},
$$

is an amplitude describing the decay and recurrence of the initial state $\left|\psi_{0}\right\rangle$. A formula of this form captures in general all quantum decay processes and has been studied in many different contexts, in particular, it is formally identical to the decoherence factor (see eq. (2)).

We can easily estimate which change in the parameter $\lambda, \Delta=\lambda_{1}-\lambda_{0}$, may lead to such anomalous relaxation processes. To do so, recall that the mean value $\overline{\mathscr{E}}_{1}$ of the energy distribution for $\lambda_{1}$ is simply related with the one at $\lambda_{0}$ through its derivative, $\mathscr{E}_{0}^{\prime}$, and the difference $\Delta: \overline{\mathscr{E}}_{1}=\mathscr{E}_{0}+\Delta \mathscr{E}_{0}^{\prime}$.

Figure 4 shows results for three quenches in the SU(1,1) model with $2 N_{b}+N_{a}=2000$ (thus $N_{a}$ even). The initial state is identified with the ground state at $\lambda=\lambda_{0}=1.5$ and the respective final parameter value $\lambda_{1}$ is written separately in each panel. In the upper row of panels we present the values $\left|c_{i}\right|^{2}$ versus the energy eigenvalue $E_{1 i}$. Note that the number of points is so large here that the scatter plots look like continuous 

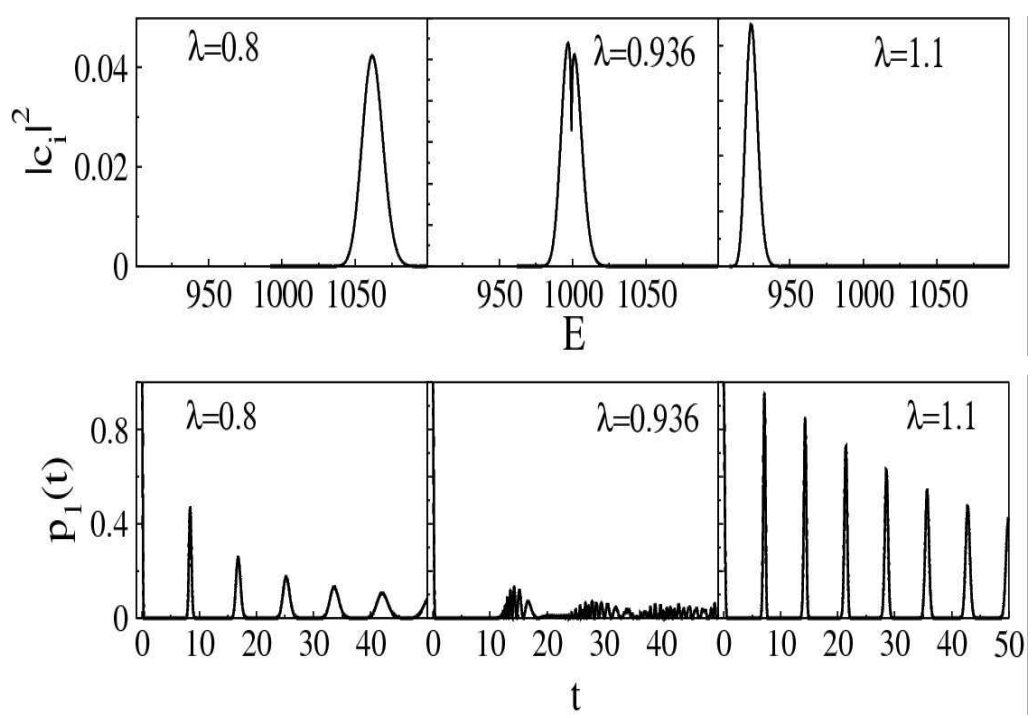

FIGURE 4. Energy distributions and survival probabilities for three quantum quenches in the SU(1,1) model $(M=2000)$. Upper row: the energy distribution of probabilities $\left|c_{i}\right|^{2}$, see Eq. (8). Lower row: the survival probability from Eq. (8).

curves. The panels from left to right correspond to a quench above, at, and below the critical energy, which for the present setting coincides with $E_{c}=1000$. While for both noncritical quenches (left and right panels) the distribution of $\left|c_{i}\right|^{2}$ exhibits just a single peak centered at energy $\bar{E}_{1}$ depending on the value of $\lambda_{1}$, the critical quench to the final value $\lambda_{1}=0.936$ (middle panel) leads to a more complex distribution. In this case we observe a double peak structure in the plot of $\left|c_{i}\right|^{2}$, the peak-separating minimum being localized exactly at the ESQPT energy.

In the lower row of panels in Fig. 4 the survival probability $p_{0}(t)$ is shown as a function of time for the three quenches discussed above. Again, similar patterns are observed for both noncritical quenches (left and right panels). In these cases, the survival probability exhibits regular damped oscillations. For the critical quench (middle panel), the survival probability behaves differently than for the noncritical cases. The quick initial decay is followed just by small random oscillations in the region $p_{0}(t) \approx 0$, avoiding the slowly damped recurrences present in the other panels. This type of dynamics is connected with the above-discussed modified form of the energy distribution shown in the upper panels of Fig. 4.

In summary, we have proved that the quantum relaxation process after a QQ is strongly distorted when the energy introduced into the system, through the sudden change of a parameter of the Hamiltonian, leads the system to a ESQPT region of the spectrum.

\section{CONCLUSIONS}

In this contribution we have studied the phenomena of Quantum Decoherence and Quantum Quench under the influence of an Excited State Quantum Phase Transition 
of second order. On one hand, we have analyzed the decoherence induced on a onequbit system by the interaction with a two-level boson environment which present QPT and ESQPT. Our main conclusion is that the decoherence is maximal when the systemenvironment coupling induces the energy gain in the environment necessary to undergo a second order ESQPT. The presence of a first order ESQPT does not enhance at all the decoherence of the qubit. On the other hand, we have used a SU(1,1) model to study the Quantum Quench phenomenon. In particular we have probed that the presence of continuous ESQPT strongly affects the quantum relaxation after a sudden change of a Hamiltonian parameter if the energy gained by the system corresponds to the position of the ESQPT.

\section{ACKNOWLEDGMENTS}

This work is presented on the occasion of Franco Iachello's 70th birthday. It has been partially supported by the Spanish Government (FEDER) under projects number FIS2011-28738-C02-01/02, FIS2009-07277, by Junta de Andalucía under projects FQM160, FQM318, P07-FQM-02962 and P07-FQM-02962, by the Spanish ConsoliderIngenio 2010 Programme CPAN (CSD2007-00042), and by the Czeck Ministry of Education (contract 0021620859).

\section{REFERENCES}

1. W. H. Zurek, Rev. Mod. Phys. 75, 715 (2003).

2. M. Nielsen and I. Chuang, Quantum Computation and Quantum Information (Cambridge University Press, Cambridge, UK, 2000).

3. E. Barouch and M. Dresden, Phys. Rev. Lett. 23, 114 (1969).

4. M. Greiner, O. Mandel, T. Esslinger, T. Hänsch, and I. Bloch, Nature 415, 39 (2002); M. Greiner, O. Mandel, T. Hänsch, and I. Bloch, ibid. 419, 51 (2002).

5. R. Gilmore and D.H. Feng, Nucl. Phys. A 301, 189 (1978); R. Gilmore, J. Math. Phys. 20, 891 (1979); R. Gilmore, Catastrophe Theory for Scientists and Engineers (Wiley, New York, 1981).

6. S. Sachdev, Quantum Phase Transitions (Cambridge University Press, Cambridge, 1999).

7. R.F. Casten, Prog. Part. Nucl. Phys. 62, 183 (2009); P. Cejnar and J. Jolie, ibid. 62, 210 (2009).

8. P. Cejnar, J. Jolie, and R.F. Casten, Rev. Mod. Phys., in press (2010).

9. M. A. Caprio, P. Cejnar, and F. Iachello, Ann. Phys 323, 1106 (2008).

10. P. Cejnar, S. Heinze, and M. Macek, Phys. Rev. Lett. 99, 100601 (2007).

11. H. T. Quan, Z. Song, X. F. Liu, P. Zanardi, and C. P. Sun, Phys. Rev. Lett. 96, 140604 (2006); F. M. Cucchietti, S. Fernandez-Vidal, and J. P. Paz, Phys. Rev. A 75, 032337 (2007); C. Cormick and J. P. Paz, Phys Rev. A 77, 022317 (2008).

12. A. Relaño, J.M. Arias, J. Dukelsky, J.E. García-Ramos, and P. Pérez-Fernández, Phys. Rev. A 78, 060102R (2008).

13. P. Pérez-Fernández. A. Relaño, J.M. Arias, J. Dukelsky, J.E. García-Ramos, Phys. Rev. A 80, 032111 (2009).

14. J. Vidal, J. M. Arias, J. Dukelsky, J. E. García-Ramos, Phys. Rev. C 73, 054305 (2006); J. M. Arias, J. Dukelsky, J. E. García-Ramos, and J. Vidal, Phys. Rev. C 75, 014301 (2007).

15. P. Pérez-Fernández. P. Cejnar, J.M. Arias, J. Dukelsky, J.E. García-Ramos, and A. Relaño, Phys. Rev. A 83, 033802 (2011).

16. E.T. Jaynes and F.W. Cummings, Proc. IEEE 51, 89 (1963); M. Tavis and F.W. Cummings, Phys. Rev. 170, 379 (1968).

17. R.H. Dicke, Phys. Rev. 93, 99 (1954). 